in spite of every care he died ten days later. For the last five days of his life he ate, in addition to his ordinary food, one or two raw kidneys.

Necropsy. - The bladder was very contracted and thickened, but otherwise it was healthy. The peritoneum showed no trace of surgical interference. Both kidneys showed advanced nephritis apostomatosa. There were bilharzia worms. The cause of death was nephritis apostomatosa chronica. The stone weighed nearly $34 \frac{1}{2}$ oz.; its largest diameters were $4 \mathrm{in}$. and 6 in. The outer layers were composed of magnesian phosphates, but no examination of the inner layer bas been made, as the stone has been presented uncut to the museum of the Royal College of Surgeons of England.

Kasr-el-Aini Hospital, Cairo.

\section{A NOTE ON THE TREATMENT OF CHOLERA.}

By MAX F. SIMON, L.R.C.P. LoND., M.R.C.S. ENG., PRINCIPAL CIVIL MEDICAL OFFICER, STRAITS SETTLEMENTS.

IN March, 1882, I was led by certain considerations to try the effect of nitro-glycerine in averting death in the collapse of cholera, and in the first few cases in which I administered the drug the success which followed was, as has been so often the case with various cholera remedies, very remarkable. On subsequent occasions, however, when I tried the remedy a like success did not follow-that is, as regards the recovery of patients-though the usual effect on the pulse was always produced. I think that most practitioners who have seen anything of cholera will agree with me that the majority of deaths from it do not occur from collapse, but from a condition supervening on collapse. The patient may recover from the diarrhoea and from the collapse which follows it-in fact, his internal temperature may rise to normal or a little above it, and the urinary secretion may return to some extent, but on about the third day of his illness he will fall into a kind of torpid condition of mind, with injected conjunctivæ and possibly flushed face, and in a day or two he will die, often comatose, apparently from a condition simulating a passive form of pneumonia. This state is, I believe, said to be dependent on a condition of the blood called "tarry blood," which means, I take it, disorganisation of the red blood corpuscles, which become inefficient as carriers of oxygen. In collapse nitro-glycerine will restore the pulse and external temperature and, provided that the blood be in a sufficiently good state, the patient will probably recover; but if the blood be much altered, though temporary apparent improvement will follow each dose of nitro-glycerine, such improvement will be only temporary and apparent and the patient will certainly die. It is evident that to do good in these cases something more is needed, and it has occurred to me that if oxygen could in addition be supplied to the blood by artificial means a line of treatment might be laid down with a good prospect of success, and it seems to me that the desired end could be gained by intravenous injection. I have not had an opportunity of making any experiments in this connexion for some time ; but I shall certainly do so should occasion arise, and I wish now to put forward for the consideration of any practitioners who have, or may have, opportunities of treating cases of cholera the line of treatment that I have indicated, and to suggest that probably the fluids best suited for the purpose of the intravenous injection will be found in solutions of permanganate or chlorate of potash, and especially the former of these substances.

During a former outbreak of cholera in England treatment by intravenous injection of saline solutions was extensively, and, I believe, with fair success, tried at the London Hospital, and it is to institutions where the staff is numerous that one must look for the trial of treatment which it takes considerable time and trouble to manage. In the Eastexcept, perhaps, in some large cities or towns-in the event of an outbreak of cholera the practitioner on the spot generally finds his hands so full that he has barely time to superintend all the necessary arrangements and cannot, as a rule, prosecute any experimental inquiry without neglecting patients in other ways. The injections at the London Hospital were made, I believe, with the intention of replacing tine volume of the blood and of supplying the lost salts, especially the chlorides, and it is possible that the success which resulted occurred in cases of simple collapse, without much blood deterioration. The cases which improved at first and afterwards proved fatal seem to point to this.

It is more than possible that some better means of supply. ing oxygen may be devised. I merely now suggest the methods that seems to me to point in the right direction. would also ask whether there may not be some analogy between the condition (I believe "tarry") of the blood which follows the bites of certain snakes, in the treatment of which permanganate of potash has been found useful, and the con dition of the blood which obtains in cholera. Nitro-glycerine may be given most conveniently by dropping two or three drops of a 1 per cent. solution of the drug in rectified spirit on the tongue and repeating as required, whilst noting the effect on the pulse, which should show itself in a few minutes If the case is one that will do well one or two doses will probably be sufficient ; if not, I have found that the supervention of headache after several doses indicates that the drug has acted to its utmost, and that it is useless to press it any further.

Some years ago, subsequently to 1882, attention was callec in one of the medical journals to the employment of nitrite of amyl in cholera collapse. This, however, did not apparently give such success as it at first appeared to promise, probably for the same reason that nitro-glycerine alone dic not always succeed.

Nitro-glycerine is now so commonly used and is so easily to be obtained that few dispensaries are without it, but in Singapore, in 1882, I had to make it, and as it is possible that others may be in a similar position it may be useful to publish the following easy and safe method of manufacture, which took from "Watts' Dictionary of Chemistry." In a conica" dispensing measure-glass mix half an ounce of pure nitric (free from nitrous) acid and one ounce of pure sulphuric acid; place the measure in a basin of water containing ice and so reduce the temperature of the mixture to between $60^{\circ}$ and $70^{\circ} \mathrm{F}$; then pour in gradually two drachms of pure glycerine, stirring al the time, and taking care that the temperature does not rise above $78^{\circ} \mathrm{F}^{\mathrm{I}}$ Oily drops will soon appear on the surface of the liquid, and when they have appeared and the glycerine is all poured in pour the whole, with constant stirring, into a large measure-glass containing about forty ounces of cold water. The nitro-glycerine sinks to the bottom and can be obtained by decanting the water; it must be washed in successive portions of water, shaking it up in each, until the water ceases to have an acid reaction. The last drops of water can be removed by a glass syringe ; they should on no account be sucked up by a pipette, for if any of the drug gets into the mouth the headache that follows is most intense The nitro-glycerine thus prepared, when precipitated, is in the form of heary, oily looking, somewhat opalescent drops, which coalesce at the bottom of the water. It can be dissolved in one hundred times its bulk of rectified spirit and is then ready for use.

\section{TOTAI, SUPPRESSION OF URINE IN A \\ PATIENT SEVENTY-FOUR YEARS OF} AGE; NEPHROTOMY; RECOVERY.

$$
\begin{gathered}
\text { BY JAMES W. FRASER, M.D., C.M. EDIN. } \\
\text { ALFRED PARKIN, M.S. LOND., F.R.C.S. }
\end{gathered}
$$

SUCCESSFUL cases of nephrotomy for complete anuria are so rarely met with that it seems to be worth while to record the following case.

A married woman aged seventy.four had enjoyed good health up to 1890 , but in that and in the succeeding year she had a short illness the chief features of which were attacks of shivering, accompanied by a rise in temperature; there was no albuminuria or hæmaturia. Her confinements, seren in all, had not been severe, nor were they followed, so far as she could remember, by any inflammatory attacks. In June of this year she began to suffer from irregular micturition, on some days passing little or no urine and on others a large

1 The best way to manage this is to stir with the thermometel if it is one that is graduated on the glass through a sufficient range temperature ; if the thermometer be an urdinary one in a rooden frane the wood below the $60^{\circ}$ mark can be cut away. 